\title{
CONSUMO E UTILIZAÇÃO DE ALIMENTO POR Spodoptera frugiperda (J. E. Smith, 1797) (Lepidoptera: Noctuidae) EM DUAS TEMPERATURAS
}

\author{
Consumption and utilization of food by Spodoptera frugiperda \\ (J. E. Smith, 1797) (Lepidoptera: Noctuidae) at two different temperatures \\ Gustavo Rossato Busato ${ }^{1}$, Anderson Dionei Grützmacher ${ }^{2}$, \\ Mauro Silveira Garcia ${ }^{2}$, Fabrizio Pinheiro Giolo ${ }^{3}$, Sandro Daniel Nörnberg ${ }^{3}$
}

\begin{abstract}
RESUMO
Conduziu-se este trabalho com o objetivo de determinar, mediante medidas de consumo e utilização de alimento, a temperatura mais adequada para a criação de Spodoptera frugiperda (J. E. SMITH, 1797) em dieta artificial. Foram individualizadas 50 lagartas em recipientes de vidro de $2,5 \mathrm{~cm}$ de diâmetro x $8,5 \mathrm{~cm}$ de altura, mantidos a $25 \pm 1^{\circ} \mathrm{C}$ e $28 \pm 1^{\circ} \mathrm{C}$, com umidade relativa de $70 \pm 15 \%$ e fotofase 14 horas. Foram determinados, com base em matéria seca, o peso seco da lagarta no máximo desenvolvimento, do alimento consumido e das fezes eliminadas, e calculados os índices nutricionais: taxa de consumo relativo (RCR), taxa metabólica relativa (RMR), taxa de crescimento relativo (RGR), digestibilidade aproximada (AD), eficiência de conversão do alimento ingerido (ECI), eficiência de conversão do alimento digerido (ECD) e custo metabólico (100-ECD). Considerando que o peso das lagartas no máximo desenvolvimento foi similar nas duas temperaturas, a $28^{\circ} \mathrm{C}$ houve maior ECI e ECD e um menor 100-ECD e, conseqüentemente, as lagartas de $S$. frugiperda consumiram uma menor quantidade de alimento, reduzindo a duração da fase larval. Assim, em laboratório, a temperatura de $28^{\circ} \mathrm{C}$ é a mais adequada para manutenção de $S$. frugiperda em dieta artificial.
\end{abstract}

Termos para indexação: Insecta, dieta artificial, índices nutricionais, lagarta-do-cartucho.

\begin{abstract}
The aim of this work was to determine the most suitable temperature for rearing Spodoptera frugiperda (J. E. SMITH, 1797) in artificial diet, by means of measuring food consumption and utilization. Fifty larvae were individualized in glass containers of $2.5 \mathrm{~cm}$ in diameter $\mathrm{x} 8.5 \mathrm{~cm}$ in height in climate chamber, at $25 \pm 1^{\circ} \mathrm{C}$ and $28 \pm 1^{\circ} \mathrm{C}$ temperature with $70 \pm 15 \%$ relative humidity and 14 hours of photophase. Based on dry matter, were determined the dry weight of the larvae at maximum development, of the food consumed and of the feces eliminated, and the nutritional ratios were calculated: relative consumption ratio (RCR), relative metabolic ratio (RMR), relative growth ratio (RGR), approximate digestibilidade (AD), efficiency of conversion of ingested food (ECI) and the efficiency of conversion of digested food (ECD). Considering that weight of larvae in maximum development was similar in two temperatures, at $28^{\circ} \mathrm{C}$ were a larger ECI and ECD and lower 100-ECD, consequently, the larvae of $S$. frugiperda consumed a lower amount of food reducing the duration of larval phase. Thus, in laboratory, the temperature of $28^{\circ} \mathrm{C}$ demonstrated to be the most appropriate for rearing of $S$. frugiperda in artificial diet.
\end{abstract}

Index terms: Insecta, artificial diet, nutritional index, fall armyworm.

(Recebido para publicação em 5 de maio de 2003 e aprovado em 22 de novembro de 2004)

\section{INTRODUÇÃO}

A lagarta-do-cartucho do milho Spodoptera frugiperda (J. E. SMITH, 1797) (Lepidoptera: Noctuidae) é uma das mais importantes pragas da cultura do milho no Brasil (CRUZ, 1995; GRÜTZMACHER et al., 2000; GALLO et al., 2002). O ataque pode ocorrer desde a fase de plântula até as fases de pendoamento e espigamento do milho. As lagartas pequenas raspam o limbo foliar das folhas mais novas, danificando, a seguir, as folhas centrais da re- gião do cartucho, o qual pode ser totalmente destruído. Em ataques tardios, as lagartas podem ser encontradas na espiga destruindo palha e grãos (ÁVILA et al., 1997). Há relatos de que as reduções na produção de milho causadas por esse inseto atingem $34 \%$, com perdas que podem variar conforme a fase de desenvolvimento da planta atacada, com a cultivar utilizada, local de plantio e mesmo entre áreas adjacentes, segundo as práticas agronômicas adotadas (CRUZ, 1995).

1. Engenheiro Agrônomo, Doutorando do Programa de Pós-graduação em Fitossanidade da FAEM/UFPel - Departamento de Fitossanidade, Faculdade Agronomia Eliseu Maciel - Caixa Postal 354 - 96010-900 - Pelotas, RS.

2. Engenheiro Agrônomo, Dr., Professor do Departamento de Fitossanidade da FAEM/UFPel.

3. Acadêmico do Curso de Agronomia da FAEM/UFPel, Bolsista BIC FAPERGS e estagiário, respectivamente. 
O controle da lagarta-do-cartucho tem sido realizado, basicamente, pela aplicação de inseticidas e pela utilização de cultivares resistentes (GASSEN, 1996; GRÜTZMACHER et al., 2000; GALLO et al., 2002). Por causa do alto custo sócio-econômico dos inseticidas e da dificuldade na obtenção de plantas resistentes e que sejam produtivas, houve a necessidade de encontrar alternativas eficientes, economicamente viáveis e ecologicamente corretas. Nesse contexto, tem sido proposto o manejo integrado de pragas (MIP), que associa os conhecimentos tanto do ambiente como da dinâmica populacional da espécie-alvo e utiliza todos os métodos e técnicas apropriadas de forma tão compatível quanto possível para manter a população da praga em níveis abaixo daqueles capazes de causar dano econômico.

Para que o MIP seja plenamente utilizável, é necessário que se conheça muito bem a cultura visada e, obviamente, as características bioecológicas das pragas a ela relacionadas (KOGAN, 1998). Entretanto, é imprescindível que se conheçam não só as características biológicas das pragas, mas também como mantê-las em condições de laboratório.

No Brasil, poucos são os estudos visando à adequação das técnicas de criação de $S$. frugiperda em laboratório. Ferraz (1982) comparou o desenvolvimento biológico desse noctuídeo em diferentes temperaturas em dieta à base de feijão e levedura de cerveja. Nalim (1991) estudou o consumo e utilização de alimento na mesma dieta anteriormente citada e em alimento à base de feijão, levedura de cerveja e germe de trigo. Souza et al. (2001) analisaram o efeito das temperaturas de 25 e $30^{\circ} \mathrm{C}$ no desenvolvimento dessa praga em dieta composta por esses ingredientes.

De modo geral, a faixa ótima de temperatura para o desenvolvimento e atividade dos insetos situa-se entre $15^{\circ} \mathrm{C}$ e $38^{\circ} \mathrm{C}$. Dentro dessa amplitude, a temperatura influencia, entre outros fatores, a velocidade de desenvolvimento, que é maior em condições mais elevadas. A temperatura mais favorável para o desenvolvimento de $S$. frugiperda é $25^{\circ} \mathrm{C}$, porém, em condições térmicas mais elevadas, normalmente ocorre uma redução da população desse inseto (FERRAZ, 1982). Segundo Singh (1983), em criações massais, busca-se reduzir o ciclo dos insetos, mantendo-se alta a viabilidade.

Nas últimas décadas, os pesquisadores têm dado maior atenção às exigências nutricionais quantitativas, considerando as variações do alimento e as pressões seletivas que o meio exerce sobre uma população e que resultam em adaptação para consumo e utilização de alimento (SLANSKY JUNIOR, 1982; SLANSKY JUNIOR e SCRIBER, 1985). Assim, a quantidade e qualidade do alimento consumido por uma lagarta afetam a taxa de crescimento, tempo de desenvolvimento, peso final, dispersão e sobrevivência e, em outros casos, a atividade dos adultos, como a fecundidade, fertilidade e dispersão (SCRIBER e SLANSKY JUNIOR, 1981; SLANSKY JUNIOR, 1982; PARRA, 1991).

Até o momento, não há informação inerente ao efeito da temperatura no aproveitamento alimentar de dieta à base de feijão, levedura de cerveja, germe de trigo, farelo de soja e caseína, visando a definir a melhor condição térmica para criação da praga em laboratório. Diante do exposto e considerando que uma variação na fonte alimentar poderá afetar as exigências nutricionais quantitativas dos insetos, com o presente trabalho objetivou-se determinar, mediante medidas de consumo e utilização de alimento a 25 e $28^{\circ} \mathrm{C}$, a temperatura mais adequada para a criação de $S$. frugiperda em dieta artificial.

\section{MATERIAL E MÉTODOS}

O experimento foi desenvolvido no Laboratório de Biologia de Insetos, Departamento de Fitossanidade (DFs), Faculdade de Agronomia "Eliseu Maciel" (FAEM), Universidade Federal de Pelotas (UFPel), Capão do Leão - RS.

As lagartas de $S$. frugiperda foram coletadas na cultura do milho no município de Pelotas - RS, sendo em laboratório mantidas na dieta modificada de Greene et al. (1976), ou seja, à base de feijão, levedura de cerveja, germe de trigo, farelo de soja e caseína. A metodologia de criação foi a mesma descrita por Parra (2001).

Individualizaram-se 50 lagartas recém-eclodidas provenientes da criação de laboratório em tubos de vidro de fundo chato de 2,5 cm de diâmetro x $8,5 \mathrm{~cm}$ de comprimento, que foram acondicionados em câmaras climatizadas reguladas a $25 \pm 1^{\circ} \mathrm{C}$ e $28 \pm 1^{\circ} \mathrm{C}$, UR de 70 $\pm 15 \%$ e fotofase de 14 horas.

Ao atingirem o último ínstar, as lagartas foram retiradas dos tubos de criação, mortas por congelamento e transferidas para estufa a $55-60^{\circ} \mathrm{C}$, por 96 horas, juntamente com a sobra da dieta e fezes existentes, visando a obter o peso do alimento consumido e o ganho de peso das lagartas. Por ser ínfima a massa corporal do inseto, essa foi desprezada (considerada zero), sendo registrado somente o seu peso no final do período de alimentação $(\mathrm{T})$ para a determinação do ganho de peso (B).

Paralelamente, separou-se uma alíquota com 10 tubos, com dieta e sem lagarta, visando à determinação do peso seco inicial da dieta (Af), essencial para o cálculo dos índices de consumo e utilização de alimento. 
Para determinação dos índices de nutrição quantitativa da fase larval, adotou-se a metodologia proposta por Waldbauer (1968) e modificada por Scriber e Slansky Junior (1981). Para o cálculo desses índices, foram utilizados os seguintes parâmetros: T: duração do período de alimentação (dias); Af: peso do alimento fornecido ao inseto (g); Ar: peso da sobra do alimento fornecido ao inseto (g), após T; F: peso das fezes produzidas (g) durante T; B: ganho de peso pelas lagartas (g) durante $\mathrm{T} ; \overline{\mathrm{B}}$ : peso médio das lagartas (g) durante $\mathrm{T}$; I: peso do alimento ingerido (g) durante T; I - F : alimento assimilado (g) durante T; $\mathrm{M}=$ (I - F) - B: alimento metabolizado durante o período de alimentação.

Foram determinados os índices de consumo e utilização de alimento, mediante as seguintes fórmulas:

- Taxa de consumo relativo (g/g/dia) - RCR $=\frac{\mathrm{I}}{\overline{\bar{B}} \cdot \mathrm{T}}$

- Taxa de crescimento relativo (g/g/dia) - RGR $=\frac{B}{\bar{B} \cdot T}$

- Taxa metabólica relativa (g/g/dia) - RMR $=\frac{M}{\bar{B} \cdot T}$

- Digestibilidade aproximada (\%) - AD $=\frac{\mathrm{I}-\mathrm{F}}{\mathrm{I}} \times 100$
- Eficiência de conversão do alimento ingerido (\%) $\mathrm{ECI}=\frac{\mathrm{B}}{\mathrm{I}} \times 100$

- Eficiência de conversão do alimento digerido (\%) $\mathrm{ECD}=\frac{\mathrm{B}}{\mathrm{I}-\mathrm{F}} \times 100$

- Custo metabólico (\%) = 100 - ECD

O delineamento experimental utilizado foi inteiramente casualizado, sendo cada lagarta considerada uma repetição. As análises estatísticas foram realizadas pelo programa "Genes" (CRUZ, 2001) e as médias, comparadas pelo teste de Tukey $(\mathrm{P} \leq 0,05)$.

\section{RESULTADOS E DISCUSSÃO}

A quantidade de alimento ingerido e de fezes produzidas foi maior na temperatura de $25^{\circ} \mathrm{C}$, não havendo diferença significativa para o ganho de peso das lagartas de $S$. frugiperda em função da temperatura (Figura 1).

A quantidade de alimento assimilado (I-F), que se refere à parte do alimento ingerido que foi utilizada pelo inseto para conversão em biomassa e/ou energia para metabolismo, e a quantidade de alimento metabolizado (M), que representa a quantidade de alimento utilizada na forma de energia metabólica, foram maiores na temperatura de $25^{\circ} \mathrm{C}$ (Figura 2).

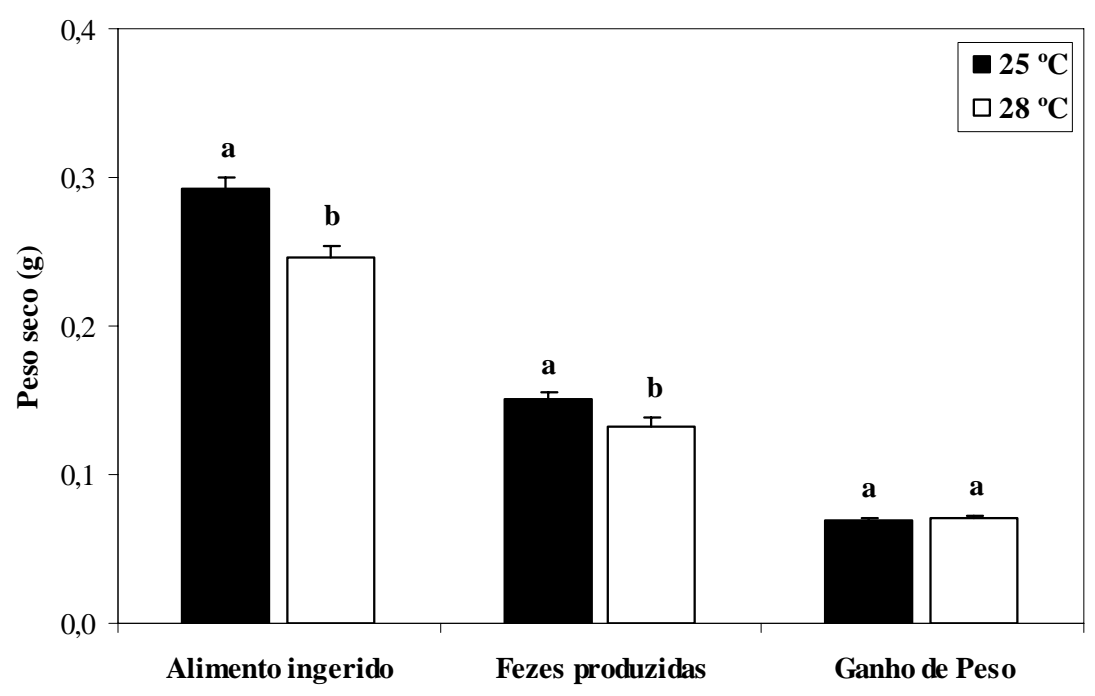

FIGURA 1 - Peso (g) do alimento ingerido, fezes produzidas e ganho de peso ( $\pm \mathrm{EP})$ por $S$. frugiperda, criada em dieta artificial a $25 \pm 1^{\circ} \mathrm{C}$ e $28 \pm 1^{\circ} \mathrm{C}$, UR de $70 \pm 15 \%$ e fotofase de 14 horas. Médias seguidas pela mesma letra não diferem entre si pelo teste de Tukey $(\mathrm{P} \leq 0,05)$. 


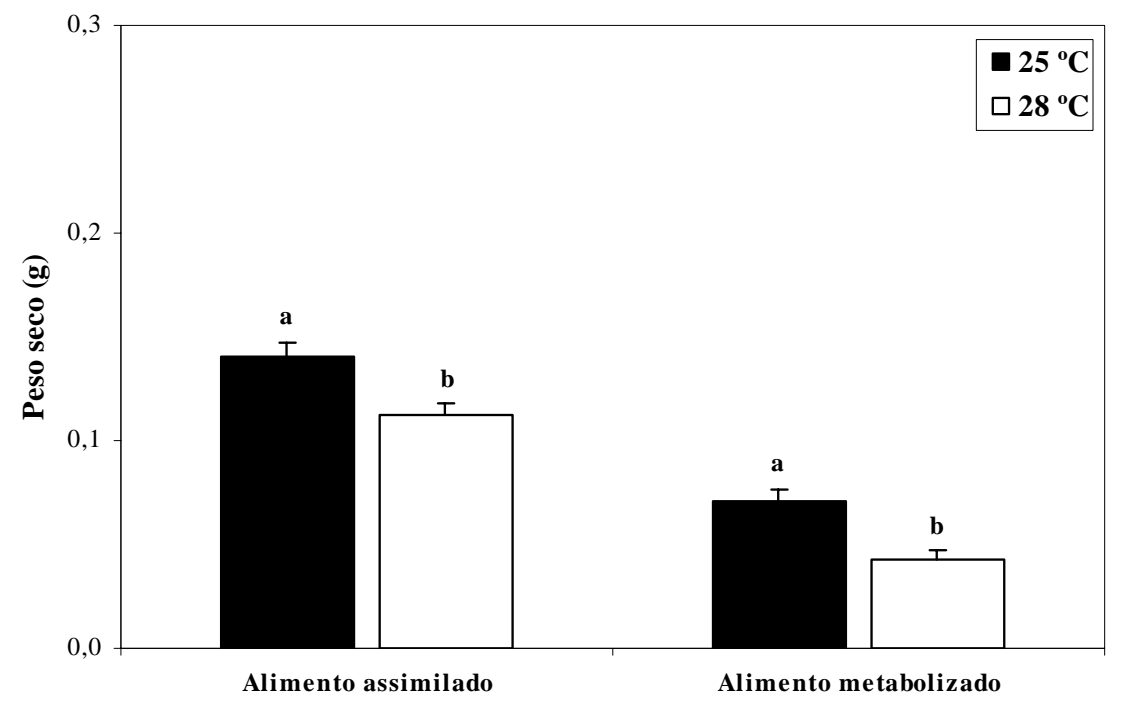

FIGURA 2 - Peso (g) do alimento assimilado e metabolizado ( \pm EP) por $S$. frugiperda, criada em dieta artificial a $25 \pm 1^{\circ} \mathrm{C}$ e $28 \pm 1^{\circ} \mathrm{C}$, UR de $70 \pm 15 \%$ e fotofase de 14 horas. Médias seguidas pela mesma letra não diferem entre si pelo teste de Tukey $(\mathrm{P} \leq 0,05)$.

A duração da fase larval foi afetada pela temperatura, sendo maior a $25^{\circ} \mathrm{C}$. Com relação aos índices nutricionais, não houve diferença significativa para a taxa de consumo relativo (RCR) em função da temperatura. A $25^{\circ} \mathrm{C}$, houve uma maior taxa metabólica relativa (RMR), que representa a quantidade de alimento gasto em metabolismo por grama de peso corpóreo, ao passo que a $28^{\circ} \mathrm{C}$, houve uma maior taxa de crescimento relativo (RGR), que indica o ganho de biomassa em relação ao peso (Tabela 1).

A digestibilidade aproximada (AD), índice que representa a porcentagem do alimento ingerido que foi efetivamente assimilado pelo inseto, bem como a mortalidade das lagartas não diferiram nas duas temperaturas. As lagartas de $S$. frugiperda criadas a $28^{\circ} \mathrm{C}$ apresentaram maior eficiência de conversão do alimento ingerido (ECI), que representa a porcentagem do alimento ingerido pelo inseto e transformado em biomassa, bem como maior eficiência de conversão do alimento digerido (ECD), que representa a porcentagem do alimento digerido convertido em biomassa. Nessa condição térmica, houve um menor custo metabólico (100-ECD), que indica a porcentagem do alimento metabolizado em energia para manutenção da vida (Tabela 1).

Os parâmetros e índices nutricionais obtidos para S. frugiperda encontram-se próximos aos valores relatados por Nalim (1991) em dieta à base de feijão e levedura de cerveja e em dieta à base de feijão, levedura de cerveja e germe de trigo, bem como àqueles obtidos por $\mathrm{Ng}$ et al. (1993) e Chang et al. (2000) em dieta à base de germe de trigo e caseína e por Souza et al. (2001) em dieta à base de feijão, levedura de cerveja e germe de trigo a 25 e $30^{\circ} \mathrm{C}$.

Constata-se que as lagartas não alteraram o ganho de peso (B), em função da temperatura. A $28^{\circ} \mathrm{C}$, em virtude de os insetos terem sido mais eficientes (maior ECI e ECD), houve um encurtamento da fase larval (T) e uma menor ingestão de alimento (I), resultando em maior taxa de crescimento (RGR). Já a $25^{\circ} \mathrm{C}$, em virtude da menor eficiência de conversão do alimento ingerido (ECI) e digerido (ECD), na tentativa de atingir o potencial fisiológico máximo (B), os insetos apresentaram uma resposta compensatória, prolongando a duração da fase larval (T) e aumentando a quantidade de alimento ingerido (I), tendo como conseqüência uma maior taxa metabólica relativa (RMR). Essa alteração nos processos fisiológicos é relatada por Slansky Junior (1982), Slansky Junior e Scriber (1985) e Slansky Junior e Rodriguez (1987).

Considerando incontestável em criações massais o binômio "redução do ciclo dos insetos $\mathrm{x}$ alta viabilidade", a melhor temperatura para aproveitamento alimentar é a de $28^{\circ} \mathrm{C}$. Resultados discrepantes aos obtidos foram relatados por Ferraz (1982), tendo constatado que $25^{\circ} \mathrm{C}$ foi a condição mais favorável para $S$. frugiperda, e por Souza et al. (2001), que verificaram um melhor desenvolvimento desse inseto a $30^{\circ} \mathrm{C}$, embora tenha 
BUSATO, G. R. et al.

TABELA 1 - Duração da fase larval, índices nutricionais e mortalidade ( \pm EP) de $S$. frugiperda criada em dieta artificial a $25 \pm 1{ }^{\circ} \mathrm{C}$ e $28 \pm 1^{\circ} \mathrm{C}$, UR de $70 \pm 15 \%$ e fotofase de 14 horas.

\begin{tabular}{|c|c|c|}
\hline \multirow{2}{*}{$\begin{array}{l}\text { Duração, Índices Nutricionais } \\
\text { e Mortalidade }\end{array}$} & \multicolumn{2}{|c|}{ Temperatura } \\
\hline & $25^{\circ} \mathrm{C}$ & $28^{\circ} \mathrm{C}$ \\
\hline Duração (dias) & $13,61 \pm 0,226$ a & $11,28 \pm 0,108 b$ \\
\hline RCR (g/g/dia) & $0,3097 \pm 0,009$ a & $0,3106 \pm 0,011 \mathrm{a}$ \\
\hline RMR (g/g/dia) & $0,0757 \pm 0,006 \mathrm{a}$ & $0,0541 \pm 0,006 \mathrm{~b}$ \\
\hline RGR (g/g/dia) & $0,0736 \pm 0,002 \mathrm{~b}$ & $0,0891 \pm 0,003 \mathrm{a}$ \\
\hline $\mathrm{AD}(\%)$ & $47,7864 \pm 1,230 \mathrm{a}$ & $45,7348 \pm 1,498 \mathrm{a}$ \\
\hline ECI (\%) & $23,9405 \pm 0,380 b$ & $29,1069 \pm 0,534 \mathrm{a}$ \\
\hline ECD (\%) & $51,6286 \pm 2,116 b$ & $65,6936 \pm 2,132 \mathrm{a}$ \\
\hline 100-ECD (\%) & $48,3714 \pm 2,116$ a & $34,3064 \pm 2,132 b$ \\
\hline Mortalidade (\%) & $24,0 \pm 6,101 \mathrm{a}$ & $16,0 \pm 5,237 \mathrm{a}$ \\
\hline
\end{tabular}

RCR: Taxa de consumo relativo; RMR: Taxa metabólica relativa; RGR: Taxa de crescimento relativo; AD: Digestibilidade aproximada; ECI: Eficiência de conversão do alimento ingerido; ECD: Eficiência de conversão do alimento digerido; 100-ECD: Custo metabólico.

Médias seguidas pela mesma letra nas linhas não diferem entre si pelo teste de Tukey $(P \leq 0,05)$.

ocorrido elevada mortalidade. Tendo em vista que a quantidade e qualidade do alimento consumido na fase larval afetam, entre outros aspectos, a taxa de crescimento, tempo de desenvolvimento e peso final, a variação nos resultados desta pesquisa pode ser atribuída à fonte alimentar utilizada na criação do inseto.

Adultos de $S$. frugiperda podem apresentar deformações nas asas, em virtude da deficiência de ácidos graxos (linoléico e linolênico) ou mesmo da interação desses ácidos com a temperatura, principalmente quando elevada (PARRA, 2001). Dessa forma, sugere-se a realização de pesquisas por meio das quais se estude o desenvolvimento biológico desse inseto nas condições térmicas testadas.

\section{CONCLUSÃO}

Em laboratório, a temperatura mais adequada para criação de $S$. frugiperda em dieta artificial é $28^{\circ} \mathrm{C}$.

\section{AGRADECIMENTOS}

À Fundação de Amparo à Pesquisa do Estado do Rio Grande do Sul (FAPERGS), pelo apoio financeiro, e ao Laboratório de Grãos, Departamento de Ciência e Tecnologia de Alimentos, Faculdade de Agronomia "Eliseu Maciel”, Universidade Federal de Pelotas, pelo auxílio concedido.

\section{REFERÊNCIAS BIBLIOGRÁFICAS}

ÁVILA, C. J.; DEGRANDE, P. E.; GOMEZ, A. S. Insetos pragas: reconhecimento, comportamento, danos e controle. In: Milho: informações técnicas. Dourados: EMBRAPA-CPAO, 1997. p. 157-170. (Circular técnica, 5).

CHANG, Y. M. et al. Influence of whorl region from resistant and susceptible corn genotypes on fall armyworm (Lepidoptera: Noctuidae) growth and development. Journal of Economic Entomology, Lanham, v. 93, n. 2, p. 477-483, 2000.

CRUZ, C. D. Programa Genes: aplicativo computacional em genética e estatística. Viçosa: UFV, 2001. $648 \mathrm{p}$.

CRUZ, I. A lagarta-do-cartucho na cultura do milho. Sete Lagoas: EMBRAPA-CNPMS, 1995. 45 p. (Circular técnica, 21). 
FERRAZ, M. C. V. D. Determinação das exigências térmicas de Spodoptera frugiperda (J.E. Smith, 1797) (Lepidoptera: Noctuidae) em culturas de milho. 1982. 81 f. Dissertação (Mestrado) - Escola Superior de Agricultura "Luiz de Queiroz", Universidade de São Paulo, Piracicaba, 1982.

GALLO, D. et al. Entomologia agrícola. 3. ed. Piracicaba: FEALQ, 2002. v. 10, 920 p.

GASSEN, D. N. Manejo de pragas associadas à cultura do milho. Passo Fundo: Aldeia Norte, 1996. 134 p.

GREENE, G. L.; LEPLA, N. C.; DICKERSON, W. A. Velvetbean caterpillar: a rearing procedure and artificial medium. Journal of Economic Entomology, Lanham, v. 69, n. 4, p. 488-497, 1976.

GRÜTZMACHER, A. D.; MARTINS, J. F. da S.; CUNHA, U. S. da. Insetos-pragas das culturas do milho e sorgo no agroecossistema de várzea. In: PARFITT, J. M. B. Produção de milho e sorgo em várzea. Pelotas: EMBRAPA Clima Temperado, 2000. p. 87-102.

KOGAN, M. Integrated pest management: historical perspective and contemporary development. Annual Review of Entomology, Palo Alto, v. 43, p. 243-270, 1998.

NALIM, D. M. Biologia, nutrição quantitativa e controle de qualidade de populações de Spodoptera frugiperda (J.E. Smith, 1797) (Lepidoptera: Noctuidae) em duas dietas artificiais. 1991. $150 \mathrm{f}$. Tese (Doutorado) - Escola Superior de Agricultura "Luiz de Queiroz", Universidade de São Paulo, Piracicaba, 1991.

NG, S. S.; DAVIS, F. M.; REESE, J. C. Southwestern corn borer (Lepidoptera: Pyralidae) and fall armyworm (Lepidoptera: Noctuidae): comparative developmental biology utilization. Journal of Economic Entomology, Coolege Park, v. 86, p. 394-400, 1993.
PARRA, J. R. P. Consumo e utilização de alimento por insetos. In: PANIZZI, A. R.; PARRA, J. R. P. (Eds.). Ecologia nutricional de insetos e suas implicações no manejo de pragas. São Paulo: Manole, 1991. p. 9-65, 359 p.

PARRA, J. R. P. Técnicas de criação de insetos para programas de controle biológico. 3. ed. Piracicaba, FEALQ, 2001. 134 p.

SCRIBER, J. M.; SLANSKY JUNIOR, F. The nutritional ecology of immature insects. Annual Review of Entomology, Palo Alto, v. 26, p. 183-211, 1981.

SINGH, P. A general purpose laboratory diet mixture for rearing insects. Insect Science, [S.l.], v. 4, p. 357362, 1983.

SLANSKY JUNIOR, F. Insect nutrition: an adaptationist's perspective. Florida Entomologist, Gainesville, v. 65, n. 1, p. 45-71, 1982.

SLANSKY JUNIOR, F.; RODRIGUEZ, J. G. Nutritional ecology of insects, mites, spiders, and related invertebrates: an overview. In: ___ Nutritional ecology, of insects, mites, spiders, and related invertebrates. New York: John Wiley \& Sons, 1987. p. 1-69.

SLANSKY JUNIOR, F.; SCRIBER, J. M. Food consumption and utilization. In: KERKUT, G. A.; GILBERT, L. I. (Eds.). Comprehensive insect physiology, biochemistry and pharmacology. Pergamom: [s.n.], 1985. v. 4, p. 165-211.

SOUZA, A. M. L.; ÁVILA, C. J.; PARRA, J. R. P. Consumo e utilização de alimento por Diatraea saccharalis (Fabr.) (Lepidoptera: Pyralidae), Heliothis virescens (Fabr.) e Spodoptera frugiperda (J.E. Smith) (Lepidoptera: Noctuidae) em duas temperaturas. Neotropical Entomology, Londrina, v. 30, n. 1, p. 11$17,2001$.

WALDBAUER, G. P. The consumption and utilization of food by insects. Advances in Insect Physiology, New York, v. 5, p. 229-288, 1968. 